\title{
XPS for probing the dynamics of surface voltage and photovoltage in GaN
}

\author{
Hikmet Sezen ${ }^{\mathrm{a}}$, Ekmel Ozbay ${ }^{\mathrm{b}}$, Sefik Suzer ${ }^{\mathrm{a}, *}$ \\ a Department of Chemistry, Bilkent University, 06800 Ankara, Turkey \\ ${ }^{\mathrm{b}}$ Departments of Physics and Electrical and Electronics Engineering, Bilkent University, 06800 Ankara, Turkey
}

\section{A R T I C L E I N F O}

\section{Article history:}

Received 10 February 2014

Received in revised form 14 June 2014

Accepted 14 June 2014

Available online 20 June 2014

\section{Keywords:}

Charging dynamics

Surface photovoltage

XPS

GaN

Doping

\begin{abstract}
A B S T R A C T
We describe application of two different data gathering techniques of XPS for probing the dynamics of surface voltage and surface photovoltage (SPV) developed in microseconds to seconds time-domain, in addition to the conventional steady-state measurements. For the longer (seconds to milliseconds) regime, capturing the data in the snapshot fashion is used, but for the faster one (down to microseconds), square wave (SQW) electrical pulses at different frequencies are utilized to induce and probe the dynamics of various processes causing the surface voltage, including the SPV, via the changes in the peak positions. The frequency range covers anywhere from $10^{-3}$ to $10^{5} \mathrm{~Hz}$ for probing changes due to charging (slow), dipolar (intermediate), and electronic (fast) processes associated with the external stresses imposed. We demonstrate its power by application to $\mathrm{n}$ - and $\mathrm{p}-\mathrm{GaN}$, and discuss the chemical/physical information derived thereof. In addition, the method allows us to decompose and identify the peaks with respect to their charging nature for a composite sample containing both n- and p-GaN moieties.
\end{abstract}

(c) 2014 Elsevier B.V. All rights reserved.

\section{Introduction}

GaN is a wide band-gap compound semiconductor with superior optoelectronic properties utilized in numerous devices for high power light emitting diode and sensor applications [1]. Surface and defect structures of the materials used completely determine many of the device performance, hence control over these properties are of utmost importance. Surface photovoltage (SPV) [2,3], is one of the properties measured to evaluate the quality and the performance of such materials and devices, and has recently been combined with microscopic techniques such as the Kelvin Probe (KP) [4,5]. SPV measurements relate electrical information to band structure, as well as to the nature of surface moieties, and most importantly to the nature of impurities and defects. However, even though a superb (sub-micron) lateral resolution is achievable, the KP is based on electrical measurements, hence does not have chemical specificity. On the other hand, X-ray Photoelectron Spectroscopy (XPS), which has also been used for probing SPV, provides excellent chemical information, in addition to the electrical potentials developed on surface structures, although the precision for measuring the latter is much lower $(\sim 20 \mathrm{meV})$ when compared to that of KP measurements $(\sim 1 \mathrm{meV})[2]$.

\footnotetext{
* Corresponding author. Tel.: +90 312 2901476; fax: +90 3122664068.

E-mail address: suzer@fen.bilkent.edu.tr (S. Suzer).
}

Earlier SPV measurements were mostly synchrotron based due to the needed brightness of the source [6-10], but combined with Xray microfocusing and fast accumulation tools, the new generation commercial XP spectrometers can also provide critical information about such materials and devices $[11,12]$. In a recent publication we reported on capturing of the transient surface photovoltage in $\mathrm{n}$ - and $\mathrm{p}-\mathrm{GaN}$ by XPS where new information about the mechanism of the transients formed by illumination with a $\sim 50 \mathrm{~mW}$ violet (405 nm) laser was brought out and discussed [13]. However, faster measurements are not possible in this mode, therefore, we had to introduce other modulation techniques [14-19].

XPS is a widely used chemical analysis technique for probing chemical and physical composition of surface structures. In conventional data gathering mode the sample is grounded, and the position as well as the intensity of the peaks are recorded in a static fashion [20]. Although several reports have appeared in the literature related to dynamical XPS measurements in the two extreme ends, very fast (nanoseconds to attoseconds) [21-24], and very slow (minutes to hours) [25-27]. The intermediate domain (microseconds to seconds) has been overlooked. The very fast measurements require either synchrotron facilities and/or specialized instrumentation which are not easily accessible to many researchers. The intermediate domain is important especially for testing and improving performance of various materials and devices used for charge storage, photovoltaics, ferroelectrics, chemical and biochemical sensing, etc. Recently, our group and others have reported 
through various publications, the basic principles and techniques for implementing such measurements employing very simple modification to conventional instruments [28-39].

Herein, we report such XPS measurements on n- and p-GaN samples, covering time intervals from kilo-seconds $\left(10^{-3} \mathrm{~Hz}\right)$ down to microsecond $\left(10^{6} \mathrm{~Hz}\right)$, and discuss the results obtained pertaining to electrical properties of the sample as reflected by the binding energy shifts of the Ga core-levels. Some of the measurements included in the present paper have already been published in our previous papers, but they are reproduced here for the sake of clarity and to provide a continuous flow of the presentation $[13,40]$. The next section describes the experimental details and is followed by our results and discussions. We finish the presentation by our conclusions, to be followed by acknowledgements and references.

\section{Experimental}

GaN samples were grown on double polished c-plane sapphire by low-pressure MOCVD (AIX 200/4 RF-S). The Mg doped p- and Sidoped n-GaN samples have conductivities of 0.8 and $58 \mathrm{~S}^{-1} \mathrm{~cm}^{-1}$, respectively. A Thermo Fisher K-Alpha electron spectrometer with monochromatic AlK $\alpha$ X-rays is used for XPS analysis, which is slightly modified for imposing external voltage stress (D.C. or A.C.) to the sample during data acquisition. The spectrometer is also equipped with a low energy flood-gun facility for charge neutralization, utilizing only electrons or both electrons and $\mathrm{Ar}^{+}$ions. Sample surfaces were cleaned by sputtering with a low energy $(200 \mathrm{eV})$ $\mathrm{Ar}^{+}$ion beam for avoiding damage by the $\mathrm{Ar}^{+}$ions, until the $\mathrm{C} 1 \mathrm{~s}$ peak fell below detection limits. No annealing of the samples was performed after cleaning. To induce surface photovoltage a $50 \mathrm{~mW}$ $405 \mathrm{~nm}$ (violet) CrystaLaser laser is employed either in the C.W. mode, or through a chopper with a frequency range of $10^{-1}-10^{3}$. For probing the dynamics of charging/discharging properties, the data is gathered either in the fast snapshot mode with $0.1 \mathrm{~s}$ time resolution or the sample is subjected to square wave pulses (SQW) of $\pm 10 \mathrm{~V}$ amplitude with varying frequencies in the $10^{-3}-10^{5} \mathrm{~Hz}$ range using a Stanford Research System DS345 pulse generator, while the data is gathered in normal scanning mode. In general the accuracy of our measurements are better than $20 \mathrm{meV}$ in the peak positions. However, since we allow the samples to charge and discharge, through the application of the SQW modulation, the peaks are broader. Even in such case, we can still claim an accuracy of $20 \mathrm{meV}$, since this time we measure the difference between two peaks positions, which is inherently more accurate when compared to absolute peak position measurements.

\section{Results and discussions}

\subsection{Photoresponse of $n$ - and p-type GaN}

\subsubsection{Steady-state measurements}

A survey spectrum of the p-type GaN has an intense $\mathrm{Ga}_{2} \mathrm{p}_{3 / 2}$ peak at $1118.49 \mathrm{eV}$ and $3 \mathrm{~s}, 3 \mathrm{p}, 3 \mathrm{~d}$ peaks at $161.41,106.48$, and $20.15 \mathrm{eV}$, respectively as shown in Fig. 1. In addition, Ga has a number of LMM Auger lines spanning the interval from 380 to $630 \mathrm{eV}$. Unfortunately, a broad LMM Auger line of Ga at $397 \mathrm{eV}$ is overlapping with the N1s peak, so it is not possible to follow individual N1s region with our XPS instrument using AlK $\alpha$ X-ray source. We generally follow the Ga2p if we need high intensity (in the snapshot mode), but the Ga3d peak is recorded for better energy resolution.

When exposed to electromagnetic radiation, materials respond in a variety of ways depending on the wavelength, intensity, and duration of the exposure. The $\mathrm{Ga}_{2} \mathrm{p}_{3 / 2}$ spectra of the $\mathrm{n}$ - and $\mathrm{p}$ GaN samples, recorded with and without violet laser illumination, are shown in Fig. 2. The highest steady-state SPV for the n- and

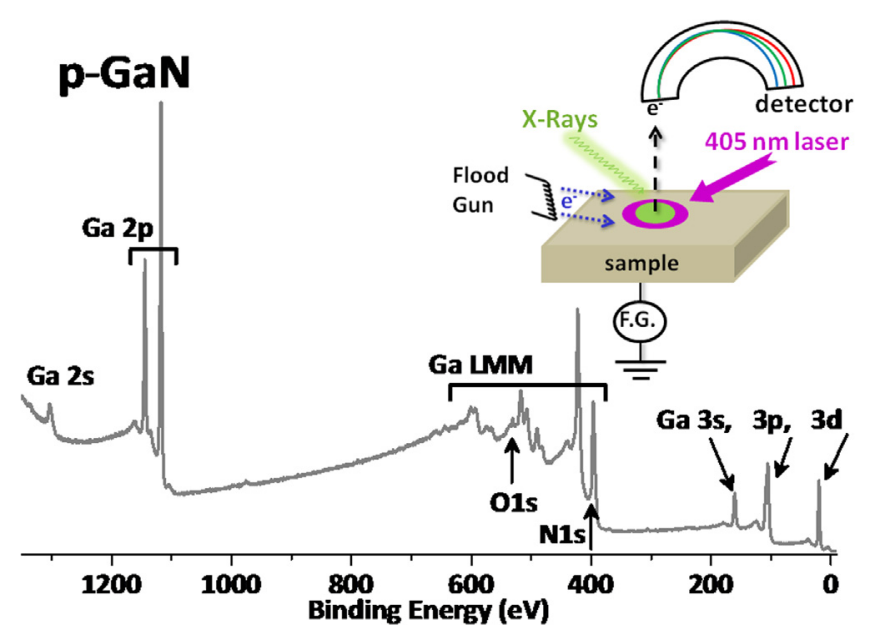

Fig. 1. A survey XPS spectrum of the p-GaN sample together with the experimental set-up.

p-GaN, are measured as $+0.15 \mathrm{eV}$ and $-0.39 \mathrm{eV}$, respectively, while the flood gun is not employed. When the flood gun is functioning, the response of especially the p-type GaN is significantly altered and provides additional information about the electrical properties of the semiconductor, as was discussed in our previous paper [13]

When the samples are introduced into the spectrometer, electrons flow from the n-type and holes from the p-type GaN so that they are charged differently, due to their sign and extent of doping. But, the charges on the surfaces are screened by band bending and by accumulation of opposite-sign charges near the surface. When light with sufficient energy is incident on the sample additional charge carriers are introduced which normally return the bands to their flat-band condition [5,10,13,40-45]. GaN has a band-gap of $3.4 \mathrm{eV}$, therefore the violet laser, which has only an energy of $3.1 \mathrm{eV}$ is not expected to cause any band-band excitation. Presence of surface states, just above the valance band, as well as defects, aided by thermal energy at room temperature must be the reason for this band-flattening. As depicted by the schematics shown in the lower panel of Fig. 2, these processes must involve an excitation of an electron and a hole from surface states into bulk for the n- and p-GaN, respectively, resulting in partial flattening of the bands.

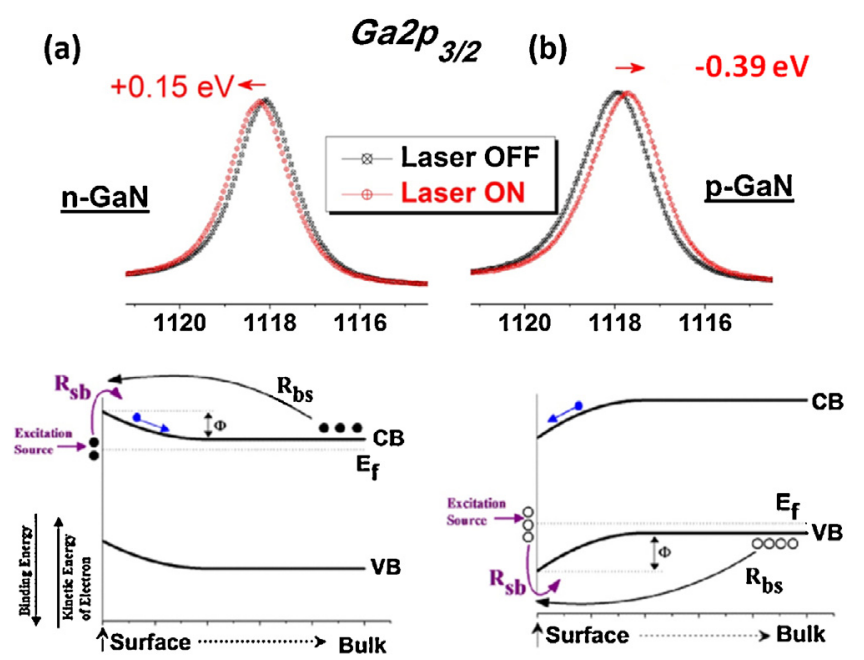

Fig. 2. Ga2 $\mathrm{p}_{3 / 2}$ region of both samples recorded without and under illumination with a C.W. Violet Laser $(405 \mathrm{~nm})$ of $\sim 50 \mathrm{~mW}$ power. The lower panels show schematically flattening of the band-bending under illumination. 


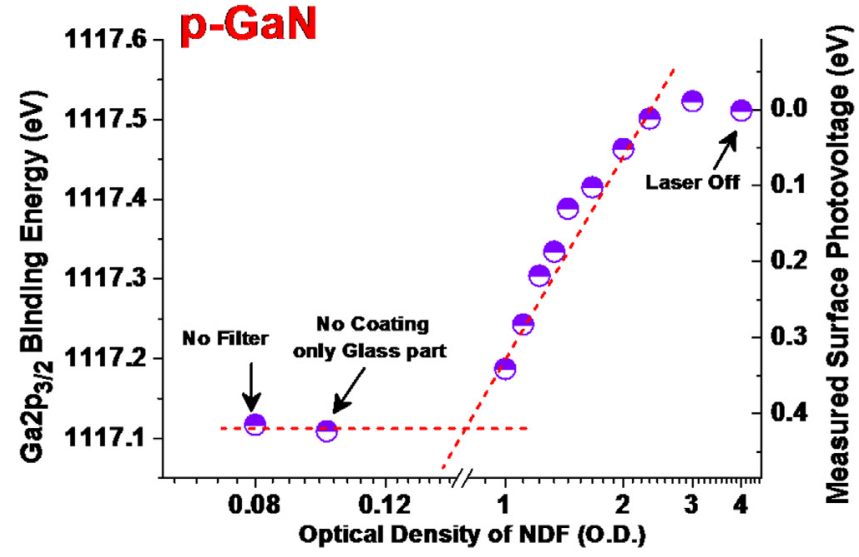

Fig. 3. Variation of the position of Ga2 $\mathrm{p}_{3 / 2}$ peak of $\mathrm{p}-\mathrm{GaN}$ with respect to the power of the illumination source, controlled by Neutral Density Optical Filters (NDF).

We have also carried out measurements by varying the intensity of the excitation source to obtain a relationship between the measured value of the SPV and the logarithm of the intensity of light as given in Fig. 3. The most important finding of these measurements, is that it reveals a saturation behavior toward the high intensity region of the photo excitement. This power dependency of the pGaN indicates that the responses we measured are mostly due to surface-states and defects' related processes, which is also in good agreement with our mechanistic explanation of the partial band flattening phenomenon involving the sub-band gap excitations.

\subsubsection{Transient measurements using the snapshot mode}

The K-Alpha spectrometer allows us to record, with reasonably high signal-to-noise ratio, a narrow spectral region with less than 0.1 s intervals. In Fig. 4(a) and (b), the time-resolved Ga2 $\mathrm{p}_{3 / 2}$ spectra are shown for the two samples while the laser is turned on and off every $50 \mathrm{~s}$, where we observe that the SPV develops in a much shorter time interval $(<0.1 \mathrm{~s})$ than we are able to measure, but it gets severely screened by the flood-gun electrons for the p-GaN. This indicates that when the flood gun is operative an additional mechanism is triggered just after the change of the state of the laser, as a result of interaction between the electrons of the flood gun and the surface of the p-GaN sample. This can be explained as follows. The p-GaN develops a certain magnitude of downward band-bending at its surface. This downward band bending at the laser OFF state provides a proper well to capture the flood-gun electrons at or near the conduction band, as schematically shown in Fig. 4(c) to cause an opposite shift to lower binding energy as expected and measured. When the state of the laser is changed to ON, the downward band-bending is immediately flattened and the well disappears. Hence, the accumulated electrons during the previous state are quickly swept away to the bulk, because now the conduction band of the bulk has many favorable energy levels for the electrons as also indicated in Fig. 4(c). This event is observed as a further shift to a higher binding energy. Moreover, the time constants of electron accumulation and electron sweeping away from the surface of the p-GaN sample are different and are 6.3 and $0.85 \mathrm{~s}$, respectively. This difference arises from the different nature of the two mechanisms. The impinging electrons take longer time to be accommodated, since each accumulated electron contributes to the build-up of the negative sheet of charge at the surface, which in turn suppresses the rate of accumulation of further negative charge. However, when the laser is turned on, the sweeping of accumulated electrons to the bulk is very effective and quick due to its large volume of the bulk compared to the surface. Note in passing that when the flood-gun is operative the SPV are smeared out, and under certain conditions, might be completely unobservable by the normal scanning mode of the spectrometer due to the time-averaged data acquisition. Only, through the careful implementation of the

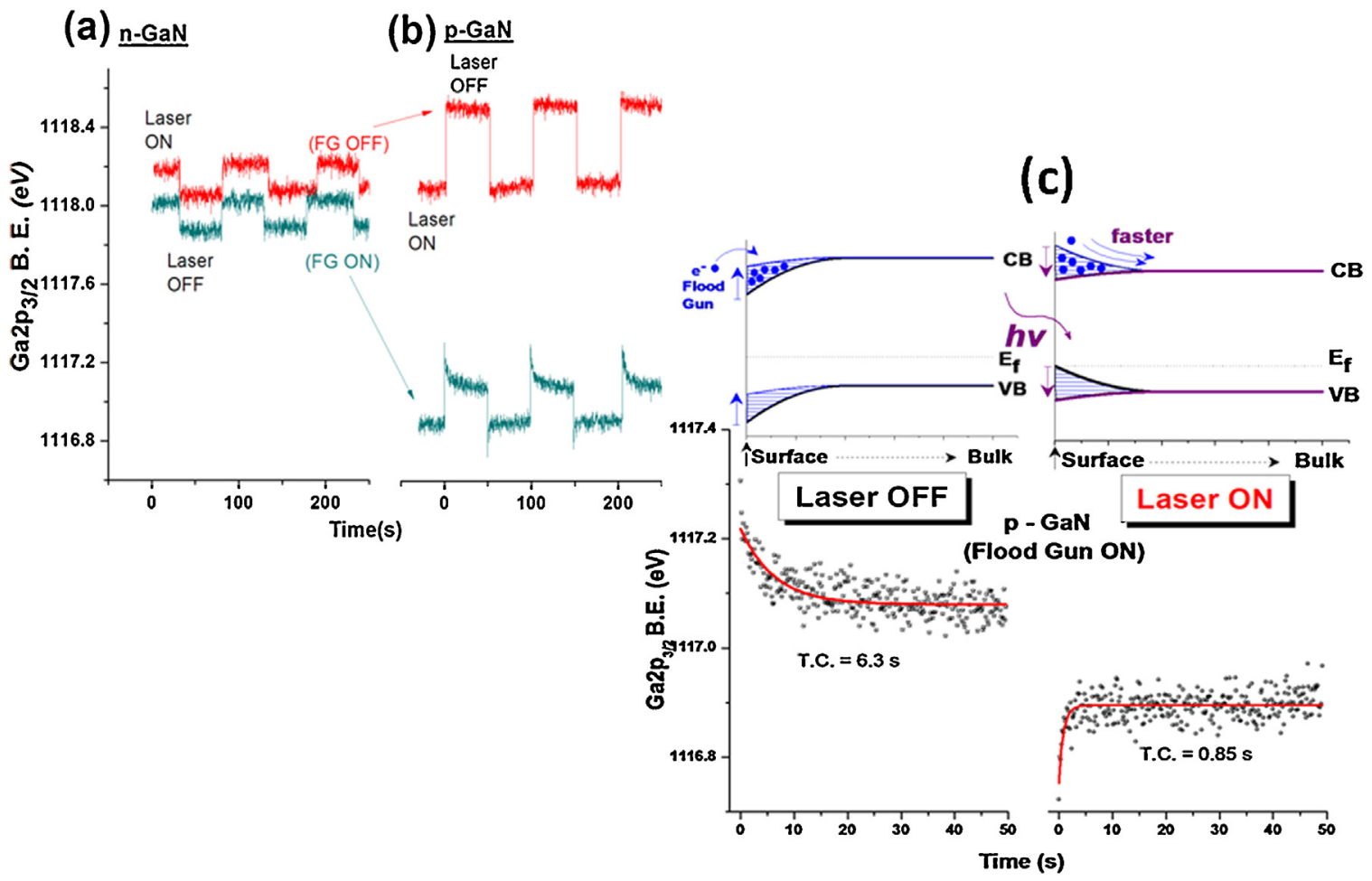

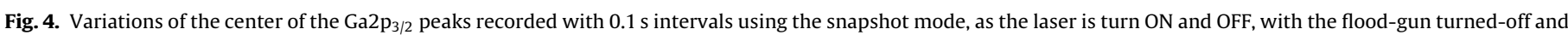
on; for (a) n-GaN, and (b) p-GaN. (c) Schematics of formation of the SPV transients for the p-GaN, and the exponential fits are given for the Laser ON and OFF periods. 


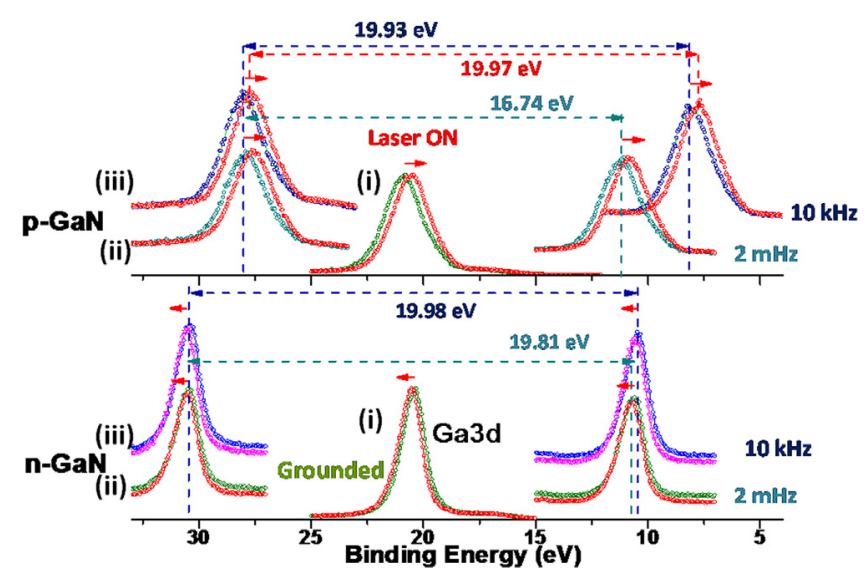

Fig. 5. Ga3d peak of the $n-$ and $p-G a N$ sample recorded; (i) grounded, and under the SQW modulation at (ii) $2 \mathrm{MHz}$, and (iii) $10 \mathrm{kHz}$ frequencies, without and under photoillumination.

snapshot mode with respect to the time windows we used were we able us to recover the full magnitude of the SPV [13].

\subsubsection{Application of SQW pulses}

Since neither the snap-shot mode nor utilization of an optical chopper can be used in the shorter microseconds regime, we make use of application of electrical voltage stresses in the form of SQW pulses. In Fig. 5, we display the Ga3d peak of the n- and the p-GaN sample recorded while the samples are grounded, as well as under $2 \mathrm{MHz}$ and $10 \mathrm{kHz}$ SQW modulations, and without and with violet laser illumination. SQW modulation of the sample results in twinning of all the peaks, since the samples experience either $+10 \mathrm{~V}$ or $-10 \mathrm{~V}$ electrical stress during the up (positive) and the down (negative) cycles, respectively. If the sample is conducting the result is trivial shifts of the peak positions to +10.00 and

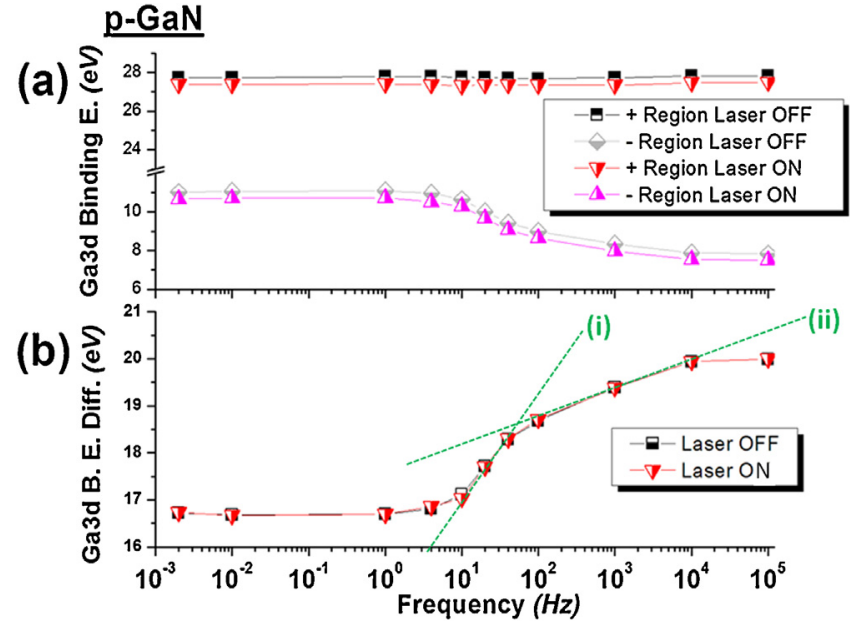

Fig. 6. Measured peak positions at $+10 \mathrm{~V}$ and $-10 \mathrm{~V}$ cycles of the Ga3d peak as a function of the frequency of the applied SQW excitation, without and under photoillumination. The lower part plots the difference between the twinned peaks.

$-10.00 \mathrm{eV}$, respectively, and with a binding energy difference measurement of exactly $20.00 \mathrm{eV}$ between them. However, if the sample is poorly conducting, a difference of less than $20.00 \mathrm{eV}$ results, since charge neutralization is different under +10 and $-10 \mathrm{~V}$ biasing cycles [14-19]. This measured negative deviation from the $20.00 \mathrm{eV}$ is larger at low frequencies since more time is allocated for the sample to charge or discharge, and approaches zero as the SQW modulation frequency increases, depending on the mechanism of the operating processes. Hence, a plot of the measured binding energy difference as a function of the applied SQW frequencies is highly informative (see below Fig. 6) [14-19].

Accordingly, as shown in Fig. 5, the binding energy difference is closer to $20.00 \mathrm{eV}$, yet slightly lower at $2 \mathrm{MHz}$, from which we

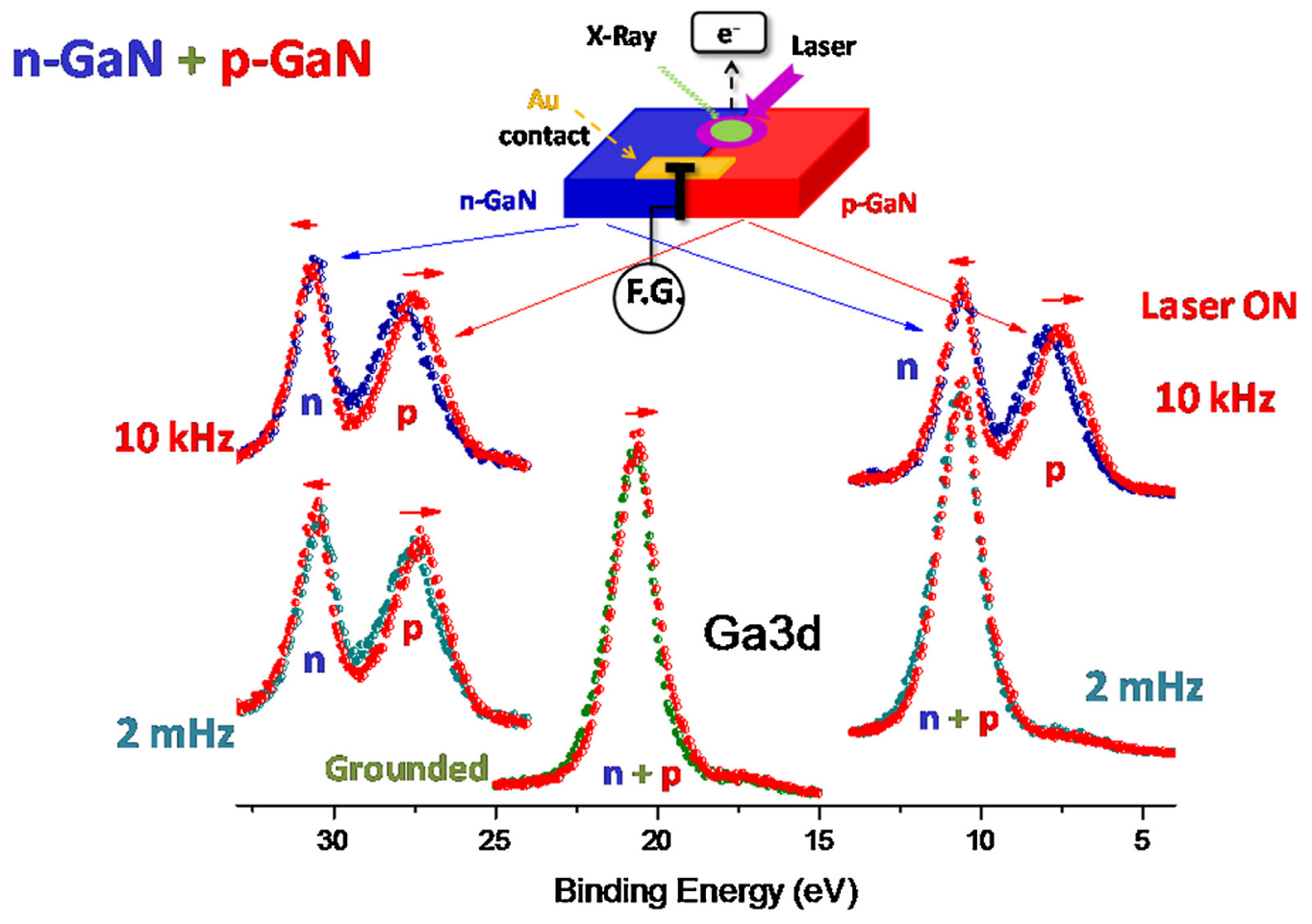

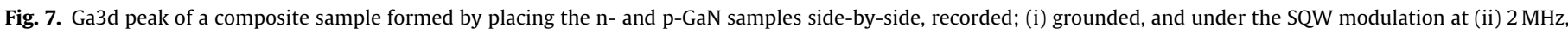
and (iii) $10 \mathrm{kHz}$ frequencies, without and under photoillumination. The inset depicts the experimental set-up. 
can now deduce that $\mathrm{n}-\mathrm{GaN}$ is much more conducting compared to the p-GaN sample, in agreement with the measured values given in the experimental section. This simple agreement is actually an important proof of the validity of our methodology, which could lead to numerous analytical applications for harvesting dielectric properties of both simple and composite surface structures $[28,46]$. In addition, the measured difference of $19.81 \mathrm{eV}$ at the lower frequency of $2 \mathrm{MHz}$ is lower than $19.98 \mathrm{eV}$ measured at $10 \mathrm{kHz}$, while the latter is within the experimental uncertainty of the theoretical value of $20.00 \mathrm{eV}$. The situation is very different for the $\mathrm{p}-\mathrm{GaN}$, which is drastically charged, and the measured value is $16.74 \mathrm{eV}$ at $2 \mathrm{MHz}$, and approaches $(19.93 \mathrm{eV})$ to the theoretical value at $10 \mathrm{kHz}$, but does not quite reach it. What one learns from these measurements is that, especially for the p-GaN, charging/discharging properties are strongly affected by a multitude of processes operative in the microseconds all the way up seconds range.

In the same figure, data recorded under light illumination are also given. For the $\mathrm{n}-\mathrm{GaN}$, the magnitude of the photoresponse is small, and is the same irrespective of the mode of data gathering, which reveals that photoresponse of the sample is much faster $(<10 \mu \mathrm{s})$ than we can measure even if we employ the SQW modulation up to the frequency of $10^{5} \mathrm{~Hz}$. The magnitude of the photoresponse of the p-GaN is much larger, and, similar to the case of the electrical charging, has multiple (slow, intermediate and fast) components, as can be deduced from the fact that even at $10 \mathrm{kHz}$ modulation, the measured binding energy difference of $19.97 \mathrm{eV}$, under illumination is larger than that $(19.93 \mathrm{eV})$ without it.

A comprehensive set of measurements for the $\mathrm{p}-\mathrm{GaN}$ is displayed in Fig. 6, where the measured positions of the twinned peaks of the Ga3d peak, as well as the difference between them are plotted as a function of frequency of the SQW pulses, both with and without C.W. violet laser illumination. The measured charging shifts are significantly altered in two domains; (i) $0.2-20 \mathrm{~Hz}$, and (ii) $0.2-10 \mathrm{kHz}$, and continue to persist even at higher frequencies. We must emphasize that all the charging shifts we have reported so far in our previous studies on various dielectric materials like $\mathrm{SiO}_{2}$, $\mathrm{CdS}$ and polymers have always been in the lower frequency range of $10^{-1}-10^{2} \mathrm{~Hz}$, and this is the first time we are able to record them in the $\mathrm{kHz}$ range, which are too fast for the usual charge compensation processes involving several cascading processes, and must somehow be related with electronic processes. Accordingly we propose that at least for the p-GaN, we have successfully demonstrated that with a combination of the snap-shot measurements and utilization of SQW pulses, XPS is able to probe and distinguish among the slow (ionic), intermediate (dipolar), and the fast (most likely due to electronic) charging processes of materials and their photoresponses.

A simple analytical extension of our method is demonstrated in Fig. 7, where we analyze a composite region consisting of both $\mathrm{n}$ - and $\mathrm{p}-\mathrm{GaN}$ samples placed side-by-side in an ad-hoc $\mathrm{p}-\mathrm{n}$ junction fashion. The Ga3d peak of the composite sample appears as a single one, when recorded with the sample is grounded, and the photoresponse is not strong enough to distinguish between the differently doped domains. However, when the SQW pulses with $10 \mathrm{kHz}$ modulation are applied, the peaks decompose to two distinct components, assignable to the $\mathrm{n}$ - or the $\mathrm{p}$ - domains, respectively. At the lower frequency the decomposition happens only for the $+10 \mathrm{~V}$ cycle, while still only one overlapping composite peak is observable in the $-10 \mathrm{~V}$ cycle. The photoresponses of the $\mathrm{n}$ - and the p- components are not as strong, but nevertheless are also indicative of the nature of the doping as can also be seen from figure.

\section{Conclusions}

By utilizing different optical and/or electrical modulation techniques, XPS spectroscopy, which is conventionally used in the static scanning fashion, can also be used for probing the dynamics of surface structures. A combination of time-resolved XPS with $0.1 \mathrm{~s}$ intervals, incorporation of SQW modulation, and use of photoillumination provides us new ways of investigating surface electronic structure and other surface properties of semiconducting and dielectric materials in a chemically specific fashion, in the microseconds to seconds time domains, which has not been reported before. One particular advantage of the method is its ability to distinguish and identify the peaks representing their doping nature for composite surface structures, using their responses to electrical, electromagnetic stresses, as well as to a combination of both.

\section{Acknowledgements}

This work was supported by the Scientific and Technological Research Council of Turkey (TUBITAK) Grant No: 212M051.

\section{References}

[1] M. Kocan, A. Rizzi, H. Luth, S. Keller, U.K. Mishra, Phys. Status Solidi B 234 (2002) 773.

[2] L. Kronik, Y. Shapira, Surf. Sci. Rep. 37 (1999) 1.

[3] D.K. Schroder, Meas. Sci. Technol. 12 (2001) R16.

[4] M. Foussekis, J.D. Ferguson, A.A. Baski, H. Morkoc, M.A. Reshchikov, Phys. B 404 (2009) 4892

[5] M.A. Reshchikov, M. Foussekis, A.A. Baski, J. Appl. Phys. 107 (2010) 113535

[6] J.E. Demuth, B.N.J. Persson, A.J. Schell-Sorokin, Phys. Rev. Lett. 51 (1983) 2214.

[7] J.E. Demuth, W.J. Thompson, N.J. DiNardo, R. Imbihl, Phys. Rev. Lett. 56 (1986) 1408

[8] A. Schellenberger, R. Schlaf, C. Pettenkofer, W. Jaegermann, Phys. Rev. B 45 (1992) 3538.

[9] R. Schlaf, A. Klein, C. Pettenkofer, W. Jaegermann, Phys. Rev. B 48 (1993) 14242.

[10] M. Moreno, H. Yang, M. Horicke, M. Alonso, J.A. Martin-Gago, R. Hey, K. Horn, J.L. Sacedon, K.H. Ploog, Phys. Rev. B 57 (1998) 12314.

[11] H. Shpaisman, E. Salomon, G. Neher, A. Vilan, H. Cohen, A. Kahn, D. Cahen, J. Phys. Chem. C 113 (2009) 3313.

[12] S. Schafer, S.A. Wyrzgol, R. Caterina, A. Jentsy, S.J. Schoell, M. Havecker, A. KnopGericke, J.A. Lercher, I.D. Sharp, M. Stutzmann, J. Am. Chem. Soc. 136 (2012) 12528.

[13] H. Sezen, E. Ozbay, O. Aktas, S. Suzer, Appl. Phys. Lett. 98 (2011) 111901.

[14] H. Sezen, G. Ertas, A. Dana, S. Suzer, Macromolecules 40 (2007) 4109.

[15] S. Suzer, H. Sezen, A. Dana, Anal. Chem. 80 (2008) 3931.

[16] S. Suzer, H. Sezen, G. Ertas, A. Dana, J. Electron Spectrosc. Relat. Phenom. 176 (2010) 52.

[17] H. Sezen, G. Ertas, S. Suzer, J. Electron Spectrosc. Relat. Phenom. 178-179 (2010) 373.

[18] H. Sezen, S. Suzer, J. Vac. Sci. Technol. A 28 (2010) 639.

[19] H. Sezen, S. Suzer, Surf. Sci. Lett. 604 (2010) L159.

[20] D. Briggs, M.P. Seah, Practical Surface Analysis, 2nd ed., Wiley, Chichester, 1999

[21] M. Marsi, R. Belkhou, C. Grupp, G. Panaccione, A. Taleb-Ibrahimi, L. Nahon, D. Garzella, D. Nutarelli, E. Renault, R. Roux, M.E. Couprie, M. Billardon, Phys. Rev. B 61 (2000) R5070.

[22] M. Marsi, M.E. Couprie, L. Nahon, D. Garzella, R. Bakker, A. Delboulbe, D. Nutarelli, R. Roux, B. Visentin, C. Grupp, G. Indlekofer, G. Panaccione, A. TalebIbrahimi, M. Billardon, Nucl. Instrum. Methods Phys. Res. A 393 (1997) 548.

[23] M. Marsi, M.E. Couprie, L. Nahon, D. Garzella, T. Hara, R. Bakker, M. Billardon, A. Delboulbe, G. Indlekofer, A. Taleb-Ibrahimi, Appl. Phys. Lett. 70 (1997) 895.

[24] M. Marsi, L. Nahon, M.E. Couprie, D. Garzella, T. Hara, R. Bakker, M. Billardon, A. Delboulbe, G. Indlekofer, A. Taleb-Ibrahimi, J. Electron Spectrosc. Relat. Phenom. 94 (1998) 149.

[25] K. Hirose, M. Yamawaki, K. Torii, T. Kawahara, S. Kawashiri, T. Hattori, Appl. Surf. Sci. 237 (2004) 411.

[26] K. Hirose, H. Nohira, K. Azuma, T. Hattori, Prog. Surf. Sci. 82 (2007) 3.

[27] K. Hirose, J. Electron Spectrosc. Relat. Phenom. 176 (2010) 46

[28] S. Suzer, Anal. Chem. 75 (2003) 7026.

[29] F. Karadas, G. Ertas, S. Suzer, J. Phys. Chem. B 108 (2004) 1515

[30] S. Suzer, A. Dana, J. Phys. Chem. B 110 (2006) 19112

[31] G. Ertas, U.K. Demirok, A. Atalar, S. Suzer, Appl. Phys. Lett. 86 (2005) 183110

[32] G. Ertas, U.K. Demirok, S. Suzer, Appl. Surf. Sci. 249 (2005) 12.

[33] S. Suzer, A. Dana, G. Ertas, Anal. Chem. 79 (2007) 183.

[34] C.P. Conger, S. Suzer, Langmuir 25 (2009) 1757.

[35] H. Cohen, R. Maoz, J. Sagiv, Nano Lett. 6 (2006) 2462.

[36] H. Cohen, S.K. Sarkar, G. Hodes, J. Phys. Chem. B 110 (2006) 25508.

[37] A. Samokhvalov, R.W. Gurney, M. Lahav, S. Cohen, H. Cohen, R. Naaman, J. Phys. Chem. B 107 (2003) 4245

[38] S.K. Sarkar, G. Hodes, L. Kronik, H. Cohen, J. Phys. Chem. C 112 (2008) 6564. 
[39] H. Cohen, J. Electron Spectrosc. Relat. Phenom. 176 (2010) 24

[40] H. Sezen, S. Suzer, J. Spectrosc. Dyn. 2 (2012) 3.

41] N.J. Halas, J. Bokor, Phys. Rev, Lett. 62 (1989) 1679.

[42] J.P. Long, H.R. Sadeghi, J.C. Rife, M.N. Kabler, Phys. Rev. Lett. 64 (1990) 1158.

[43] J.P. Long, V.M. Bermudez, Phys. Rev. B 66 (2002) 121308.
[44] E.A. Kraut, R.W. Grant, J.R. Waldrop, S.P. Kowalczyk, Phys. Rev. Lett. 44 (1980) 1620

[45] S.A. Ding, S.R. Barman, K. Horn, H. Yang, B. Yang, O. Brandt, K. Ploog, Appl. Phys. Lett. 70 (1997) 2407

[46] M.T. Camci, S. Suzer, J. Vac. Sci. Technol. A 32 (2014) 021510. 